\title{
Eficiencia de mezclas para el control de nematodos a base de aceites esenciales de orégano, canela y extractos hidroalcohólicos de gobernadora
}

\section{Efficiency of mixtures for the control of nematodes based on essential oil of oregano, cinnamon and hydroalcoholic extracts of governor}

\author{
MARÍN-TINOCO, R. I ${ }^{1} \dagger^{*}$., SILVA-MARRUFO, $\mathrm{O}^{1}$., CASTAÑEDA-VENEGAS, J. A ${ }^{1}$. y SIDA \\ ARREOLA, J. P2
}

${ }^{1}$ Universidad Tecnológica de Rodeo, Departamento de Microbiología General, Carretera Federal Panamericana Km. 159.4, Colonia. ETA. C.P. 35760, Rodeo, Dgo.

${ }^{2}$ Centro de Investigación en Alimentación y Desarrollo A. C. Unidad Delicias, Chih.

ID $1^{\text {er }}$ autor: R.I. Marín-Tinoco / ORC ID: 0000-0003-4885-223X, Researcher ID Thomson, X-2101-2018, CVU CONACYT ID: 161831

ID $1^{\text {er }}$ Coautor: O. Silva-Marrufo. / ORC ID: 0000-0003-2064-5298X, Researcher ID Thomson: X-223-2018, CVU CONACYT ID: 847832

ID $2^{\text {do }}$ Coautor: J.A. Castañeda-Venegas. / ORC ID: 0000-0001-9448-9393X, CVU CONACYT ID: 949036

ID $3^{\text {er }}$ Coautor: J. P. Sida-Arreola / ORC ID: 0000-0003-3029-58X, Researcher ID Thomson: W-9323-2019, CVU CONACYT ID: 331168

\section{Resumen}

Esta investigación fue desarrollada con el fin de determinar la concentración idónea de la mezcla entre aceite esencial de orégano (AEO), Aceite de canela (AC) y una extracción hidroalcohólica de gobernadora (EHG) en el tratamiento de suelos contaminados por nematodos. El objetivo del trabajo fue identificar y eliminar nematodos presentes en el suelo; se desarrolló en el laboratorio de tecnología de agua de la Universidad Tecnológica de Rodeo. El método utilizado fue de flotación para identificar los nematodos y un simplex centroide para concentración optima. Se emplearon tratamientos orgánicos AEO, AC, y EHG para el control de nematodos sin dañar a las plantas. Donde se obtuvo la presencia de la especie de nematodo helicotylenchus, por medio de microscopia 40x (Forma de gusano vermiforme/espiral), en una muestra de suelo recolectada del invernadero Agrocemosa S.P.R de R.L de C.V. en Jalpa, Nombre de Dios Durango. Empleando el Simplex Centroide con los resultados siguientes: un modelo cuadrático DU: R$\mathrm{SQR}=85.77 \%$; ADJ; $67.99 \%$ de variables de respuestas para las mezclas de los tratamientos: donde la mezcla más eficiente fue el tratamiento 10 -AEO $0.66 \%$, EHG $0.17 \%$ y AC $0.17 \%$ con el $99 \%$ y el tratamiento 3.- AEO $0.5 \%$, EHG $0.00 \%$ y AC $0.5 \%$ con el $99 \%$ para el control de nematodos. Concluyendo que este método es eficaz en la aplicación de aceites esenciales orgánicos en la eliminación de nematodos presentes en suelos contaminados.

Orégano, Canela, Gobernadora, Aceite esencial y nematodos

\begin{abstract}
This research was developed in order to determine the ideal concentration of the mixture between oregano essential oil (AEO), Cinnamon oil (AC) and a governor hydroalcoholic extraction (EHG) in the treatment of soils contaminated by nematodes. The objective of the work was to identify and eliminate nematodes present in the soil; It was developed in the water technology laboratory of the Technological University of Rodeo. The method used was flotation to identify the nematodes and a simplex centroid for optimal concentration. AEO, AC, and EHG organic treatments were used to control nematodes without damaging the plants. Where the presence of the helicotylenchus nematode species was obtained, by means of 40x microscopy (vermiform/spiral worm form), in a sample of soil collected from the Agrocemosa S.P.R greenhouse of R.L de C.V. in Jalpa, Name of God Durango. Using the Simplex Centroide with the following results: a quadratic model DU: R-SQR $=85.77 \%$; $\mathrm{ADJ} ; 67.99 \%$ of response variables for the treatment mixtures, where the most efficient mixture was treatment 10 -AEO $0.66 \%$, EHG $0.17 \%$ and $\mathrm{AC} 0.17 \%$ with $99 \%$ and treatment 3.- AEO $0.5 \%$, EHG $0.00 \%$ and $0.5 \%$ AC with $99 \%$ for nematode control. Concluding that this method is effective in the application of organic essential oils in the elimination of nematodes present in contaminated soils.
\end{abstract}

Oregano, Cinnamon, Governor, Essential oil and nematodes

Citación: MARÍN-TINOCO, R. I., SILVA-MARRUFO, O., CASTAÑEDA-VENEGAS, J. A. y SIDA ARREOLA, J. P. Eficiencia de mezclas para el control de nematodos a base de aceites esenciales de orégano, canela y extractos hidroalcohólicos de gobernadora. Revista de Ingeniería Biomédica y Biotecnología. 2019. 3-8: 23-28

\footnotetext{
* Correspondencia del Autor (ruben.marin@utrodeo.edu.mx)

$\dagger$ Investigador contribuyendo como primer autor
} 


\section{Introducción}

La presencia y ataque de nematodos constituye grandes pérdidas económicas, por el bajo rendimiento y producción de hortalizas, los nematodos que afectan las plantas producen síntomas tanto en raíces como en el follaje de la planta, los síntomas que presentan las raíces son nudos y lesiones dentro de estas; ramificaciones excesivas de la raíz, pudriciones de la raíz que también van acompañadas de bacterias y hongos (Pacheco, 2004). Este proyecto se basa principalmente en la identificación del control de nematodos mediante el método de Flotación, también el saber usar la cantidad correcta de los tratamientos orgánicos no dañinos para el medio ambiente y el suelo, esto para que las hortalizas estén libres de desechos tóxicos que alteran el organismo humano, esto se llevará acabó en el área de invernadero y laboratorio de microbiología general de la Universidad Tecnológica de Rodeo.

\section{Fundamentación Teórica}

\section{Morfología de nematodos}

Los nematodos son animales acuáticos muy delgados que viven en la película de agua que rodea las partículas de suelo, también se encuentran en el estiércol, basura orgánica y en sedimentos de estanques, ríos y océanos, aún a profundidades de 5,000 metros en el Golfo de Vizcaya y a una densidad de un distal proximal 5 millón de nematodos por metro cuadrado (Guzmán y Castaño, 2010).

\section{Cutícula y estructuras externas}

La cutícula de los nematodos es elástica y está conformada de diferentes capas, siendo la más externa reemplazada durante el proceso de muda (Jhonson et al., 1972).

\section{El sistema digestivo de los nematodos}

La boca, así como los dientes de un mamífero y el pico de un pájaro la boca nos brinda información acerca de la alimentación del individuo. La estructura de la cavidad bucal (Estoma) y el esófago nos brinda información acerca de los hábitos alimenticios del nematodo (Perry et al., 2009).

\section{Esófago e intestino de nematodos}

El lumen del odontoestilete penetra proximalmente en el lumen del esófago y éste a su vez en el intestino. El esófago también se denominada faringe, es muscular y tiene tres glándulas en Tylenchidos o cinco glándulas en los Dorylaimidos. La estructura general de un esófago tylenchido (Borgers, 2015).

\section{Microscopia}

El estudio detallado de los componentes de células y tejidos animales o vegetales, por el tamaño que poseen, requiere el uso de instrumentos que permitan ampliar muchas veces más la imagen de las estructuras que los constituyen (Arenas, 2010).

\section{Orégano}

El orégano, (Origanum vulgare), pertenece a la familia Labiaceae, y es una planta herbácea vivaz muy aromática (Koksal et al., 2010).

\section{Gobernadora}

La gobernadora (Larrea ratia), es un arbusto perenne de los desiertos Chihuahuense, Sonorense y Mojavede Norteamérica. Los metabolitos secundarios de la resina (Entre los que destacan fenoles, lignanos y flavonoides), (Saldivar, 2003).

\section{Canela}

El aceite esencial de canela (Cinnamomum zeylanicum) es utilizado como bactericida gracias a su componente principal, el eugenol, presente en un 70-95\% (Husain et al., 1989; González, 2002).

\section{Metodología a desarrollar}

\section{Descripción del área de estudio}

El presente trabajo se desarrolló en las instalaciones de la Universidad Tecnológica de Rodeo en el municipio de Rodeo, Dgo, (Figura 1). Se localiza al centro del estado de Durango. Colinda al norte con el municipio de San Pedro del Gallo; al noreste con San Luis del Cordero; al oriente con Nazas; por el sur San Juan del Río. Su cabecera municipal se encuentra ubicada en las coordenadas $25^{\circ} 11^{\prime}$ de latitud norte y $104^{\circ} 34^{\prime}$ de longitud oeste, a una altura de 1,340 msnm. 


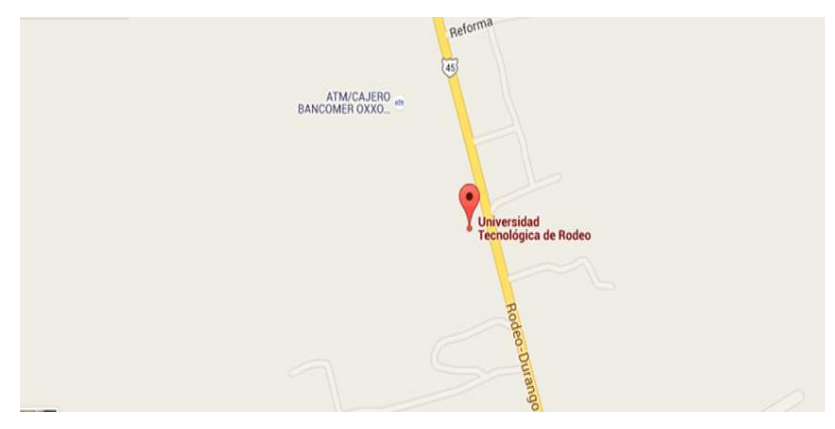

Figura 1 Localización de área de estudio Fuente: Silva, 2019

\section{Método de extracción hidroalcohólico de gobernadora}

En esta actividad se realizó las mediciones en el alcohol en diferentes proporciones de $50 \mathrm{~mL}$, $100 \mathrm{~mL}, 150 \mathrm{~mL}$ y $200 \mathrm{~mL}$, posteriormente se mezcló y se midió la cantidad correcta con agua destilada en diferente alcohol dentro de cada matraz, se introdujo las mezclas en cada una de las botellas, por lo consiguiente se depositó las hojas de gobernadora en una cantidad de $25 \mathrm{~g}$ en cada botella, para después tapar las botellas con aluminio para que no penetre la luz solar directamente cuando este en reposo.

\section{Método de extracción Arrastre de vapor}

Aceite de canela y orégano según lo reportado por Martínez, (2018).

\section{Identificación de nematodos con el método de flotación}

Para la identificación de nematodos, colocamos cada muestra en una proporción con $250 \mathrm{~g}$ por triplicado, posteriormente se tamizo la muestra por diferentes orificios por consiguiente se filtró lo restante de la muestra y se colocó en un papel filtro y finalmente se realizó la técnica de flotación.

\section{Inoculación de los tratamientos a las muestras}

En la inoculación de los diferentes tratamientos en primer lugar, se aforo a $5 \mathrm{~mL}$ de agua para poder mezclar con cada uno de los tratamientos con las medidas correspondientes y posteriormente se añadieron las mezclas en las muestras de suelo. Por lo subsecuente los análisis se realizaron tres replicas por cada tratamiento, para el cálculo de mortalidad se calculó el porciento de mortalidad igual al número de nematodos muertos entre el número total de nematodos por cien.
Y se desarrollaron por cuenta en microscopio óptico en 40X por 10 campos (Iler, 2017).

\section{Obtención de datos Método con el simplex centroide}

El Diseño Simplex-Centroide (DSC) de la Metodología de Superficie de Respuesta consiste en un triángulo que representa todo el universo de posibilidades de mezcla de los tres ingredientes evaluados (AEO, EHG y AC), (Siche, 2016). El análisis estadístico de regresión múltiple para evaluar el efecto de los ingredientes en el diseño fue realizado usando el programa Minitab 15 y requirió de un análisis de varianza $(\alpha=0.05)$, de modelos de, cuadrático, cual se construyó la superficie de respuesta para determinar los rangos de los tres ingredientes que optimizan el diseño, en la tabla 1 se muestra el diseño aleatorizado (Siche, 2016).

\begin{tabular}{|l|r|c|c|c|}
\hline Tratamiento & \multicolumn{1}{c}{ Tipo } & AEO & EHG & AC \\
\hline 1 & 0 & 0.3333 & 0.3333 & 0.3333 \\
\hline 2 & -1 & 0.6667 & 0.6667 & 0.1667 \\
\hline 3 & 2 & 0.0000 & 0.0000 & 0.5000 \\
\hline 4 & -1 & 0.1667 & 0.1667 & 0.1667 \\
\hline 5 & 1 & 1.0000 & 1.0000 & 0.0000 \\
\hline 6 & 2 & 0.5000 & 0.5000 & 0.5000 \\
\hline 7 & 1 & 0.0000 & 0.0000 & 0.0000 \\
\hline 8 & 2 & 0.5000 & 0.5000 & 0.0000 \\
\hline 9 & 1 & 0.0000 & 0.0000 & 1.0000 \\
\hline 10 & -1 & 0.1667 & 0.1667 & 0.6667 \\
\hline
\end{tabular}

Tabla 1 Diseño (Aleatorizada)

*AEO=Aceite Esencial de Orégano. ${ }^{*} \mathrm{EHG}=$ Extracto Hidroalcohólico de Gobernadora. *AC=Aceite de Canela.

\section{Resultados}

A continuación, se presentan las observaciones obtenidas en cronología: identificación de nematodos, numero de nematodos muertos, mezclas y grafica de rastreo de Cox.

\section{Identificación de nematodos con el método de flotación}

En la Figura 2 se muestra el nematodo extraído por la técnica de flotación en una muestra de suelo recolectada en el invernadero semiautomatizado en las instalaciones de la empresa Agrocemosa S.P.R de R.L de C.V. en Jalpa, Nombre de Dios Durango. 
En base a la guía de nematodologia de D.L. Coyne, J.M. Nicol y B. Claudius- Se pudo identificar por sus características morfológicas el tipo de nematodo presente en la muestra de suelo, dando como resultado la clase Helicotylenchus por medio de microscopia óptica 40X (Forma de gusano vermiforme/espiral), (Claudius-Cole, 2009).

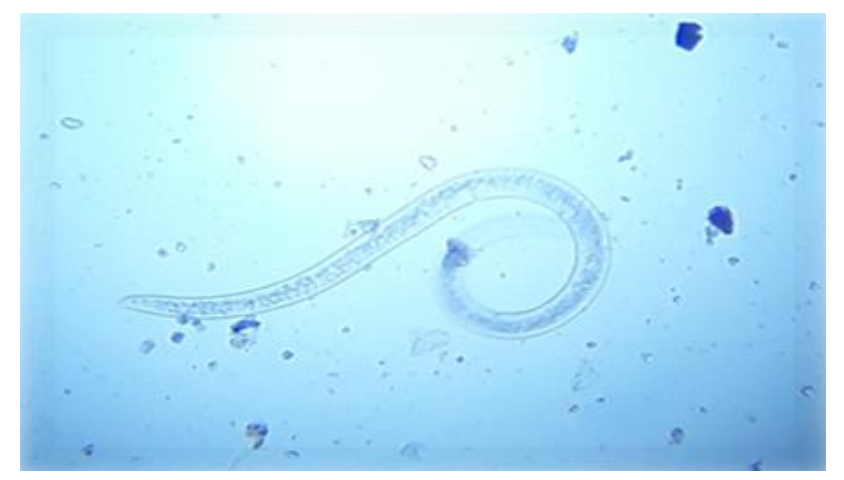

Figura 2 Nematodo Helicotylenchus, Microscopia 40x Fuente: García, 2019

\section{Mezclas del método simplex-Centroide en relación con nematodos muertos}

En la tabla 2 sé presentan los 10 tratamientos del diseño elaborado con porcentajes diferentes de AEO, EHG y AC, esta varió en el rango de 99.90 a 99.9 de la escala de evaluación de 0 a 100, lo cual sugiere que los niveles porcentuales de AEO, EHG y AC, permitieron una exploración completa del diseño, con un modelo cuadrático DU: $R-S Q R=85.77 \%$ y un ADJ; $67.99 \%$ en variables de respuesta en nematodos el tratamiento 10 con una proporción de $\mathrm{AEO}$ $0.66 \%$, EHG $0.16 \%$, AC $0.16 \%$ y el No. 3 con una proporción de AEO $0.50 \%$, EHG $0 \%, 105$ $\%$ AC evidenció el mayor porcentaje de muertes de nematodos lo que refiere una interacción positiva entre ingredientes de los componentes.

\begin{tabular}{|c|c|c|c|c|c|}
\hline Tratamiento & Tipo & AEO & EHG & $\mathbf{A C}$ & $\begin{array}{l}\text { NM } \\
\%\end{array}$ \\
\hline 1 & 0 & 0.3333 & 0.3333 & 0.3333 & 99.93 \\
\hline 2 & -1 & 0.6667 & 0.6667 & 0.1667 & 99.96 \\
\hline 3 & 2 & 0.0000 & 0.0000 & 0.5000 & 99.97 \\
\hline 4 & -1 & 0.1667 & 0.1667 & 0.1667 & 99.97 \\
\hline 5 & 1 & 1.0000 & 1.0000 & 0.0000 & 99.96 \\
\hline 6 & 2 & 0.5000 & 0.5000 & 0.5000 & 99.93 \\
\hline 7 & 1 & 0.0000 & 0.0000 & 0.0000 & 99.96 \\
\hline 8 & 2 & 0.5000 & 0.5000 & 0.0000 & 99.97 \\
\hline 9 & 1 & 0.0000 & 0.0000 & 1.0000 & 99.90 \\
\hline 10 & -1 & 0.1667 & 0.1667 & 0.6667 & 99.96 \\
\hline
\end{tabular}

Tabla 2 Diseño de mezclas y numero de nematodos muertos

*AEO=Aceite Esencial de Orégano. *EHG=Extracto Hidroalcohólico de Gobernadora. *AC=Aceite de Canela. *NM=Nematodos Muertos.
Como se muestra en la figura 3 en el punto 0 de la derecha que causa el $99.97 \%$ de mortandad, así como el punto central superior $99.97 \%$ de mortandad, como menciona Iler (2017), la concentración de 0,75\% de AE de orégano al cabo de 8 horas de exposición logró una mortalidad de nematodos de $100 \%$.

Así como Ayvar (2017), menciona extracto de canela (Qanum), lograron reducir un $66.7 \%$ el número de larvas con respecto al tratamiento control; ya que en su composición química existen compuestos como timol, carvacrol y cinamaldehido que actúan a nivel de membrana celular de nematodos.

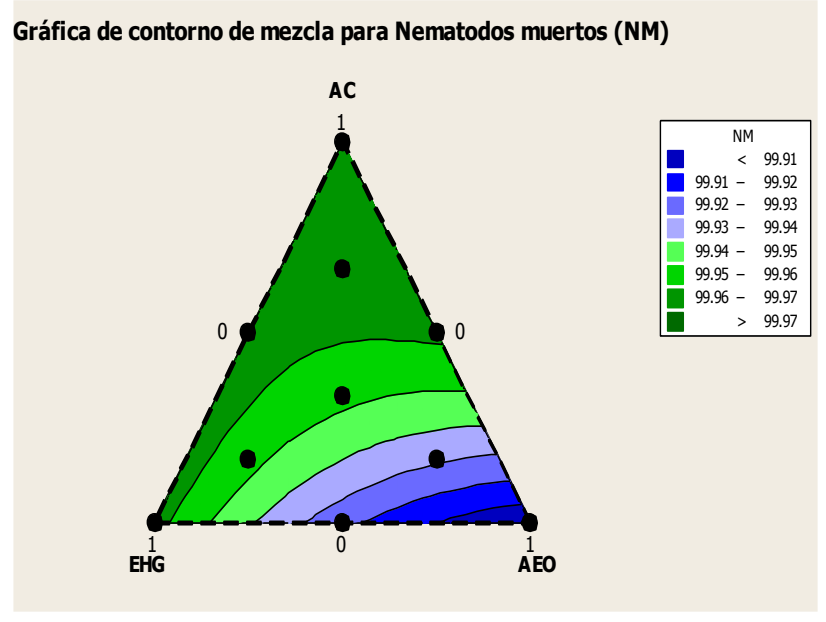

Figura 3 Diagrama de contorno de mezcla para nematodos muertos

Fuente: Marín, 2019

\section{Mezclas homogéneas de aceites esenciales}

En la siguiente grafica de rastreo de respuesta de Cox, se muestra la mezcla homogénea que fue la más eficiente para la inhibición de nematodos a base de aceite de canela con una mezcla de aceite de orégano (Figura 4). En una investigación por Ayvar-Serna et al. (2018), el extracto de canela (Qanum), se registró la mayor actividad antagónica, porque lograron reducir un $66.7 \%$ el número de larvas con respecto al tratamiento control, en el presente experimento se redujo la presencia de nematodos de $99.96 \%$ con una concentración de canela del $0.333 \%$. 


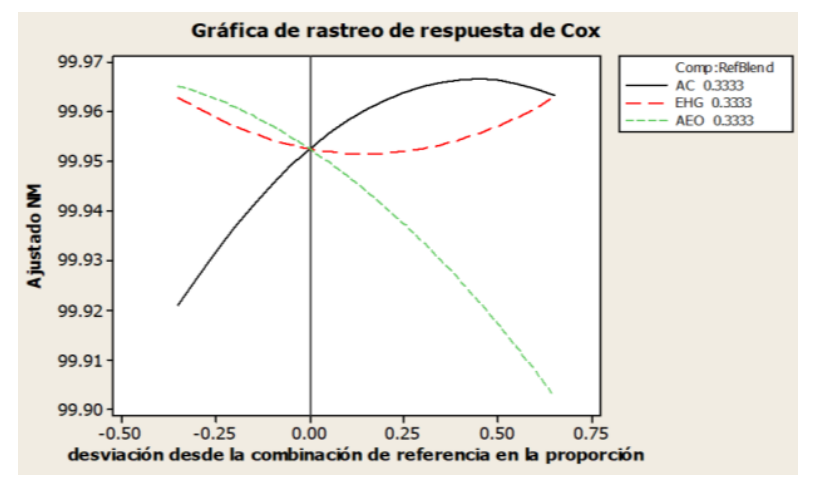

Figura 4 Diagrama de rastreo de respuesta de Cox Fuente: Marín, 2019

En cuestiones con el Extracto Hidroalcoholico de Gobernadora, se obtuvo un porcentaje de $99.96 \%$ con la mezcla 5 con $1.000 \%$, en una investigación realizado por Galarza-Tristán et al. (2018), utilizo mezclas de extractos de neem, gobernadora y calabacilla loca lo cual obtuvieron promedios de mortalidad de alrededor de $40 \%$, por lo anterior no concuerdan los presentes resultados; ya que estos extractos presentan mayores concentraciones de gobernadora a diferencia de los extractos vegetales.

\section{Agradecimiento}

Los principales autores agradecen a la Universidad Tecnológica de Rodeo, por las facilitaciones en el área de laboratorio de tecnología del agua.

\section{Conclusiones}

Mediante el diagrama grafico de diagnósticos, se utilizaron tres tratamientos principales mayoritarios en el aceite esencial de orégano, canela y una extracción hidroalcohólica de gobernadora.

Comprobando que la mezcla del aceite de orégano y canela disminuyo la severidad de Helicotylenchus presente en el suelo recolectado. El método de simplex centroide es eficaz en la aplicación de aceites esenciales orgánicos para la eliminación de nematodos presentes en suelos deteriorados.

\section{Recomendaciones}

Ensayar otros tipos de aceite esencial, con mayor concentración para la eliminación de los diferentes nematodos.
Utilizar los diferentes aceites esenciales como fúngicos orgánicos para el control de hongos en plántulas de tomate.

\section{Referencias}

Arenas, C. M. (2010). Microscopia. 1-18.

Ayvar-Serna, S., Díaz-Nájera, J.F., AlvaradoGómez, O.G., Velázquez-Milán, I., PelaezArroyo, A., y Tejada-Reyes, M.A. (2018). Actividad nematicida de extractos botánicos contra meloidogyne incognita (Kofoid y whithe) en okra (Hibiscus esculentus L. Moench). Revista de las Ciencias Biológicas y de la Salud. Universidad de Sonora. Biotecnia. V: XX. (1). 13:19, (2018).

Borgers, T. (2015). Morfologia de nematodos. curso de identificacion.

Claudius-Cole, B. (2009). Guía de campo y laboratorio. Nematologia, 1-99.

González R. (2002). Eugenol: propiedades farmacológicas y toxicológicas. Ven-tajas y desventajas de su uso. Revista Cu-bana Estomatol 39: 139-156.

Galarza-Tristán, F.E.; Aldama-Aguilera, C.; Hipólito-Cruz, G.; González-Montero, R.; Medellín-Castillo, N.A.; Bernal-Jácome, L.A. (2018). Extractos vegetales para el control de larvas de mosquitos en diferentes calidades de aguade la Ciudad de San Luis Potosí. Revista de Entomología Mexicana. 5:148-154. ISSN: 2448$475 X$.

García-Martínez, K. I. (2018). Extracción y Caracterización Fisicoquímica del Aceite Esencial del Orégano (Origanum vulgare), en la Localidad de "Héroes de México" del Municipio de Rodeo, Dgo. Revista de Simulación y Laboratorio, 5-15:7-13.

Guzmán, P. O. A. y Castaño, Z. J. (2010). Identificación de nemátodos fitoparásitos en guayabo (Psidium guajava L.), en el municipio de Manizales (Caldas), Colombia. Revista Académica Colombiana Científica. 34(130):117-125.

Husain A, Virmani P, Misra N. (1989). The major essential oil-bearing plants ofIndia. Lucknow, India: Central Instituteof Medicinal and Aromatic Plants(CSIR). 49 p. 
Iler, D. C. (2017). Evaluación de la actividad nematicida in vitro de aceites esenciales frente a Meloidogyne. Ambato, Ambato, Ecuador.

Jhonson, S. R.; Ferris, V. R. and Ferris, J. M. (1972). Nematode community structure of forest woods lots I. Relactionship bases on similarity coefficients of nematode species. J. Nematol. 4:175-183.

Koksal, O.; Gunes, E.; Orkan, O. and Ozden, M. (2010). Analysis of effective factor on information sources at Turkish oregano farms. African J. Agric. Res. 5:142-149.

Perry, R.; Moens, M. and Starr, J. (2009). Root knot nematodes. $\mathrm{CAB}$ International. London. $480 \mathrm{p}$.

Saldivar, R. H. (2003). Estado Actual del Conocimiento Sobre las Propiedades Biocidasde la Gobernadora. Revista Mexicana de FITOPATOLOGIA, 1-222.

Velasquez, L. (2016). Diseño de simplex centroide y la funcion de deseabilidad de la optimizacion de la aseptabilidad sensorial del pan dulce. Researchgate, 1-81.

Siche, R. (2016). El Diseño Simplex-Centroide y la Función de Deseabilidad en la optimización de la aceptabilidad sensorial de pan dulce enriquecido con Chenopodium quinoa. Enfoque UTE, 70-81. 